\title{
IMMUNOBIOCHEMICAL AND HAEMATOLOGICAL INDEXES OF RATS BLOOD AFTER THE REMOVAL OF SPLEEN
}

\author{
Struchko G U', Merkulova L M \\ Stomenskaya I $S^{1}$, Zahid $M^{1}$, Kayastha $P^{1}$
}

ABSTRACT

Background: The spleen is not a vitally important organ, but the removal of spleen can adversely affect the patient's health, especially that of children. Abrupt decrement in antimicrobial resistance after the splenectomy is confirmed experimentally leading to overwhelming postsplenectomic hyposplenism. Besides there are few researches in the pathogenesis and mechanisms of development of serious infectious complications after splenectomy. So in our research we studied some indexes of immunology, general laboratory and biochemical tests of blood in different terms after splenectomy.

Methods: The immunologic, general laboratory and biochemical studies were done in $3,7,15,30,45,60$ and 120 days after the splenectomy on 90 white male laboratory rats.

Findings: Splenectomy causes great changes of immunologic indexes of blood: from 7 day of the experiment there's a fall of IgG level, some increase of complement activity, the decrease of neutrophile's phagocytosis activity. In 4 months after the removal of spleen the level of IgG and IgM is almost half, the number of circulated immunocomplexes is raised three fold and the neutrophile's phagocytosis activity remains low. Besides there is a heavy haemolytic anaemia in 7 days after the splenectomy, the leukaemoid reaction of myelocyte type, the thrombohemorragic syndrome and as a result of it - the acute renal insufficiency symptoms of which disappear by 120 days after the operation.

Conclusion: The removal of spleen causes the development of accidental involution of thymus and because of it causes the development of secondary immunodeficiency, thrombohaemorrhagic syndrome and severe anaemia. It needs to have more considered approach to the splenectomy and if it can't be avoided - to initiate immunocorrection therapy at the earliest.

Key Words: Splenectomy, immunodeficiency, leukaemoid reaction, renal insufficiency.

1. Chuvash State University, Department of Functional and Laboratory Diagnostics, Russia.

Address for correspondence : Struchko GU

Universitetskaya Street, House 17, Flat 41

Cheboksary, 428034

Russia

Email: funklb@chuvsu.ru 


\section{INTRODUCTION}

Splenectomy is widely used in the hospitals, though the surgical indications to this operation have been revised during the last decade. The most common reasons for the removal of spleen are portal hypertension, autoimmune haemolytic anaemia, idiopathic thrombocytopenic purpura, spleen injury, hypoplastic conditions of haemopoiesis etc. ${ }^{1}$ The spleen is not a vitally important organ, but the removal of spleen can adversely affect the patient's health, especially that of children. And, as the practice shows, the temporary improvement of general state and good clinical and laboratory indexes change to immunodeficiency. ${ }^{2,3}$ At present, abrupt decrement in antimicrobial resistance after the splenectomy is confirmed experimentally leading to OPSI (overwhelming postsplenectomic hyposplenism) or postsplenectomic hyposplenism, symptoms of which are - decrease in immunity, possibility of development of fulminant infections, also boost of morbidity with acute and chronic viral and bacterial infections during the whole further lifetime of patient. ${ }^{2-8}$

The important role of the spleen as haemopoietic and lymphoid organ was established by the numerous researches. The functions of spleen includes active antibody and humoral mediators production, the concentration of T-suppressers, $\mathrm{T}$ helpers and the part of effector lymphocytes. ${ }^{9}$ The both stages of differentiation of antibody-producing cells from the medulla precursors and antigen dependent stage of T-lymphocytes differentiation also takes place in spleen..$^{10,11}$ The complicated structure of spleen lymphoid follicles, including thymus dependent, thymus independent and macrophage elements, establishes the favourable conditions for cells co-operation in immune response. ${ }^{12,13}$
The literary facts about the immunoactivity after splenectomy are very contradictory. In the opinion of some authors the removal of spleen causes the great disturbances of all immune system components. ${ }^{3,5,7}$ Other scientists mention that it is a temporary disturbance as a result of reaction of neuroendocrine organs on surgical stress..$^{14}$ Some scientists consider that there is no immune reaction after splenectomy at all. ${ }^{15,16}$

Most researches in immunology were done some years later after the removal of spleen, when the symptoms of the postsplenectomic hyposplenism had already appeared. Besides there are only few scientific research have been done regarding pathogenesis and mechanisms of development of serious infectious complications after splenectomy.

The purpose of this research was to study some indexes of immunology, general laboratory and biochemistry in different terms after splenectomy.

\section{MATERIAL AND METHODS}

Experiments were carried out on 90 white breedless laboratory male rats of mass $170-300 \mathrm{~g}$. The animals were kept with all the rules of animal care. The animals were divided into 3 groups: the first group of 15 rats was surveyed without influence (intact rats), the second group of 30 rats - falsely operated ones with incision along the white abdomen line on the third group of animals (45 rats) splenectomy was performed via white abdominal line incision. The operation was done under ether mask narcosis observing all aseptic and antiseptic rules. The blood was taken on 3, 7, 15, 30, 45, 60 and 120 days after the operation.

The blood indexes (the levels of erythrocytes, leukocytes, platelets, haemoglobin) were counted 
on the automatic machine (Sysmex USA). The blood smears were stained by Romanovsky-Gimsa's method and used for studying leukogram. ${ }^{17}$ The concentration of immunoglobulins in blood was determined by radial immunodiffusion in agar by Manchini. ${ }^{18}$ Besides the activity of complement system by $50 \%$ hemolysis, ${ }^{18}$ the phagocytic activity of neutrophils with latex and the calculation of phagocytic number (the average number of latex particles absorbed by one active neutrophil) and phagocytic index (the percentage of phagocytes among the counted neutrophils), the level of circulated immunocomplexes ${ }^{18}$ were also examined. The biochemical indexes of blood (urea, creatinine, uric acid, cholesterol, calcium) were determined on automatic biochemical analyzer (Dimension AR Du Pont, USA).

\section{RESULTS}

After splenectomy we observed the changes in red blood indexes (table I) as early as $3^{\text {rd }}$ day. The number of erythrocytes decreases by $20 \%$ and there is up to one third fall of the haemoglobin level. At the end of the first week after the removal of spleen, the lowest figures of erythrocytes and haemoglobin were observed as low as one fourth compared to false-operated and intact animals. In blood smears, there was significant poikilocytosis and anisocytosis. In 7 days after the operation, $75 \%$ of cases revealed 7 - 15 normoblasts per 100 cells. The level of erythrocytes and haemoglobin gradually increased, but it did not reach the initial level (at intact animals) even in 120 days after splenectomy (table I).

Table I : Haematological indexes in intact rats and changes in rat after the splenectomy 
The number of leukocytes begins to increase as early as 3 days after the operation and is 1.6 time more than normal level (table I). In leukogram, the percentage of segmented neutrophils rises due to decrease of quantity of lymphocytes and monocytes. In 7 days, the leukocytes increased by 6 times with an absolute neutrophilia. In $60 \%$ rats, leukaemoid reaction was revealed more often of myelocyte type. Sometimes, the leukocytes reaches $50-60 \times 10^{9} / 1$, while in intact rats it's usually not higher than 5$6 \times 10^{9} / 1$. Leukaemoid reaction is accompanied with the increase of neutrophils level with different degree of segmentation till the determination of the blastcells (about 1-2 cells per 100 leucocytes). During this period the monocytes are absent.

After 15 days of splenectomy, the absolute lymphocytosis with the relative neutropenia and monocytopenia on the background is marked. Leukaemoid reactions on myelocyte type are found in $20 \%$ of cases but they are not so expressed and the blast cells are not found. Leucocytes gradually decrease and reaches the level at intact and false- operated animals after 120 days. It should be marked that the quantity of lymphocytes changes like waves. The increase takes place in 45-60 days and considerably decreases in 120 days after the operation with development of absolute lymphopenia. Platelets count in rats is significantly

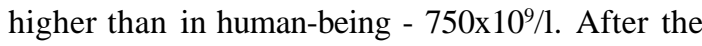
removal of spleen, significant changes of the number of platelets are marked in 15, 45 and 120 days after the operation (table I). It should be noted that 7 days after the splenectomy and further in general laboratory study of blood in rats, platelets count ranged from $300-1300 \times 10^{9} / 1$. It's possibly related with different speed of thrombohaemorhagic syndrome development as a result of erythrocytes destruction and with registration of different stages of this syndrome.

Regarding blood immunoglobulin it was marked that the changes of IgG and IgM was significant. The concentration of IgG decreases in 7 days after the removal of spleen and it persists at low rates till the end of 4 months.

Table II : The immunological indexes in intact rats and changes in rats after the splenectomy (in 3-120 days)

$*-P<0.01, * *-P<0.001$ 
The level of $\operatorname{IgA}$ also begins to decrease in 7 days, however by 30 days it returns to normal level (Table II).

The concentration of IgM increases four-fold in 3 days. But follow up showed this increase of $\operatorname{IgM}$ was transitory and in 7 days of the experiment the level of immunoglobulins returned to normal.

The essential changes in complement system are not revealed in our study (Table II).

Regarding the neutrophils phagocytosis activity it was noted that the level of basic phagocytosis significantly decreased during the first week after the operation. After the first week, both the phagocytosis index and phagocytosis number didn't change. At the same time the stimulated by prodigiosane phagocytosis during the whole period of the investigation was lower than at the control group of animals and reached to the minimum activity during the first 2 weeks after the operation.

From $15^{\text {th }}$ day onwards of the experiment, there was increase in circulated immunocomplexes by 2-3 times which sustained till the end of the research.
In blood biochemical indexes, there was marked increase of urea and creatinine levels. It increase as early as 3 days after splenectomy. Its level decreased near to normal after 15 days but still on the higher side till the end of the research

\section{DISCUSSION AND CONCLUSIONS}

The removal of spleen causes great and prolonged changes in general, biochemical and also in immunological indexes of rats blood. Some reasons of the revealed changes can be determined, but to the current knowledge, the main reason is the disturbance of function of suprarenal glands, pituitary and thymus system. Splenectomy causes the great morphological changes of suprarenal glands structure ${ }^{19}$ and at the same time increment of glucocorticoids level that caused more serious breaches of thymus function with the development of the acute thymic insufficiency. ${ }^{14,20,21}$ Another study concluded that splenectomy assists in reducing mass of thymus, decreasing the sizes of it cortex and medulla and increasing mast cells population in thymus, the growth of serotonin and histamine in all it's structures. It is known that serotonin and histamine are suppressive mediators

Table III : Indexes of blood biochemical study in intact rats and changes in rats after the splenectomy

$*-P<0.01, * *-P<0.001$ 
that reduce the development and differentiation of thymus T-lymphocytes. ${ }^{23}$ All this testifies the growth of the immature cortisone sensitive $\mathrm{T}$ lymphocytes apoptosis in thymus and the development of the accidental involution of thymus. Of course, it influences on immune status of organism as a whole. It is revealed that not only the insufficiency of cellular immunity ${ }^{24}$ but also decreases the level of immunoglobulins like IgG and $\operatorname{IgM}$. The $\operatorname{IgM}$ reduction can be explained by the most synthesis of this immunoglobulins in spleen. Precisely, the decrease in IgM is related with the inclination of splenectomized patients to infections, especially to pneumococcal infection because these antibodies are the powerful opsonisation factors acting against the bacteria with polysacharide capsule..$^{2,25}$

The changes in blood cell's phagocytic activity during early days after the operation ${ }^{3}$ ismost likely related with the depression of the ability of neutrophils. The reduction of phagocytic activity with prodigiosan is probably related with disappearance of serum polypeptide taftsine ${ }^{26}$ that is synthesised in spleen and intensifies macrophagocyte and polymorphonuclear leukocyte phagocytosis and their other functional properties. Some increase of the basic phagocytosis in 2-4 months after the operation can be explained by increased level of circulated immunocomplexes in blood. Their increase is probably related with reduction in the capture of these complexes by mononuclear phagocyte system because part of them are fixed in the spleen.

The development of leukaemoid reaction in the first 2 weeks caused by the breaches of immune and endocrine systems and is probably a compensated-protected reaction. The severity and duration this reaction depends on the character of the added infection as a result of developed postsplenectomic hyposplenism.

Another implication of splenectomy worth mentioning is the heavy anaemia developed in the first two weeks after the operation. The haemolytic nature of anaemia can not be excluded. Further research is needed to find out the whether it is due to intravascular hemolysis or the increased destruction of erythrocytes. The fact that the levels of urea, creatinine and uric acid are increased after the splenectomy indicates the development of acute renal insufficiency because of intravascular hemolysis. Its indirect confirmation can be made from the development of thrombohaemorrgic syndrome after splenectomy in rats. ${ }^{27}$

In conclusion it should be marked that the removal of peripheral immune organ resulting in the development of accidental involution of thymus leads to the development of secondary immunodeficiency, thrombohaemorragic syndrome and severe anaemia. It needs to have more considered approach to the splenectomy and if it can't be avoided - to initiate immunocorrection therapy at the earliest.

\section{REFERENCES}

1. K oushtch N L , Z hourilo I $P, K$ ononouchenko V P. S plenectomy in children. Surgery 1988; 7 : 84-88.

2. G ogin E E, R outkovskij V V. I nfectious complications in patients with blood diseases after splenectomy. C linical M edicine 1985; 5: 95- 98.

3. S amsygin $S A, D$ olgina $E N, S$ mirnov $A N, M$ artina $S \mathrm{~V}, \mathrm{~K}$ onyaeva $\mathrm{OL}, \mathrm{K}$ ostomarova $\mathrm{T} \mathrm{D} . \mathrm{I}$ mmune status in children after splenectomy due to trauma. $\mathrm{H}$ ematology and $\mathrm{T}$ ransfusiology 1985; 6: $42-47$. 
4. D ourdyev M D, P ashoutin SB.A Iteration of immunological data after splenectomy and reimplantation of spleen fragments. $B$ ulletin of E xperimental B iology and M edicine 1985; $\mathrm{V}$ ol. 99, 6: 719-720.

5. E pyfanov N S. S plenectomy and risk of serious infections devel opment in children. P ediatrics 1991; 3: 96-99.

6. K oushtch N L, Z hourilol $P, V$ alenkoY S I nfluence of splenectomy on immunological responsiveness of organism in children. C linical S urg. 1987; 6: 11-13.

7. $\quad P$ avlovskij $M P, C$ houklin $S N, O$ ryel $G L$.I nfluence of splenectomy on immunologic reactivity. S urgery 1986; 6: 136-141.

8. K rivit W. A mer. J. H aemat. 1977; 2: 193-201

9. $V$ inogradova $J E, I$ vanina $E K, S$ krjabin $A$ S. I nfluence of splenectomy on phagocytic activity of neutrophiles. T herapeutical A rchive 1983; 6: 68-71.

10. M elchers F. B iol. C ell. 1978; 32: 9-18.

11. 0 smond D J.J. R eticuloendoth. S oc. 1975; 228: $672-673$

12. A geev A K.P athology A rchieve 1976; 12: 3-11.

13. H lystova $Z S, R$ yabchikov $O P, C$ huich $N A$, S hmel yeva SP. I mmunology 1982; 3: 27-30.

14. A rion $V J$ J. I $n$ : $T$ he adsorbtion methods of detoxication and immune correction in medicine. K harkov,1982: 216-217.

15. P ougachev A G, G oryachev V V.I nfluence of splenectomy on immunological indexes in children. C linical Surg. 1983; 6: 13-16.

16. A dami $G F, S$ copinaro $N, C$ ariati $E$. L ymphology 1982; 15: 40-42.

17. R omeys $B$. T he technology of microscopy. M oscow: Foreign L iterature, 1954.

18. M edical laboratory technologies. E d. by A I $K$ arpishtchenko. $S$ anct- $P$ eterburg: I ntermedica, 1999.
19. Stomenskaya I S, S truchko G U, M ohammad $Z$ ahid. $M$ orphofunctional research of adrenal glands structures after splenectomy. I $n$ : T opical $P$ roblems of $D$ iagnostics and $T$ reatment in I nternal D iseases C linic's. C heboksary, 2000.

20. C hebotaryev VF. E ndocrine regulation of ommunogenesis. K iev: Z dorov'ja, 1979.

21. J anossy $G, B$ offil $M, T$ reidosiewiez $L$. C ellular differentiation of Iymphoid subpopulations and thier microenvironments in the human thymus. $\mathrm{I} n$ : $\mathrm{T}$ he $\mathrm{H}$ uman $\mathrm{T}$ hymus. $\mathrm{E} d$. by $H$. M ullerH ermelink. B erlin, 1986 .

22. S truchko $G U, M$ erkulova $L M, S$ ergeeva $V E$, $S$ tomenskaya $\mid S$. R esponce of bioaminecontaining rat thymus structures to experimental removal of the spleen. I mmunology 2000; 2: 13-17.

23. S ergeeva $V E, G$ ordon D S . L uminescenthystochemical characteristic of thymus monoamincontaining structures early reaction to antigen influence. $\mathrm{C}$ heboksary: $\mathrm{C}$ huvash $\mathrm{S}$ tate U niversity, 1992.

24. B atyrbekov $A$ A, M anjko V $M, R$ udneva $T$ B , $\mathrm{H}$ alatjan $\mathrm{N} \mathrm{A}$. I nfluence of splenectomy on hemopoietic stem cells differentiation and functional activity of $\mathrm{T}$ - Iymphocytes. I mmunology 1985: 5: $41-45$.

25. W eissman I L . T ransplant. R ev. 1975; 24: 159176.

26. $K$ ragballe $K, L$ anng $N$ J , S olling J. S cand. J . H ematol. 1981; 27: 271-278.

27. K ousin $M I, R$ udneva $V G, D$ ourdyev $M D$, Shimkevich $L L$. T he state of thrombocytic component of hemostasis in patients after splenectomy and reimplantation of spleen fragments. H ematology and $T$ ransfusiology 1985; 8: 26-29.

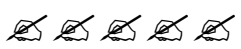

